\title{
Au-delà de l'évolution technologique : réflexion muséologique pour des cyberexpositions conséquentes et particularisées
}

Beyond the technological revolution: museological thoughts about consistent and individual cyber exhibitions

\section{Eric Langlois}

\section{OpenEdition \\ Journals}

Édition électronique

URL : http://journals.openedition.org/iss/607

DOI : $10.4000 /$ iss. 607

ISSN : 2306-4161

Éditeur

ICOM - International Council of Museums

Édition imprimée

Date de publication : 1 juin 2015

Pagination : 139-155

ISSN : 2309-1290

Référence électronique

Eric Langlois, «Au-delà de l'évolution technologique : réflexion muséologique pour des

cyberexpositions conséquentes et particularisées », ICOFOM Study Series [En ligne], 43a | 2015, mis en ligne le 06 février 2018, consulté le 21 avril 2019. URL : http://journals.openedition.org/iss/607 ; DOI : $10.4000 /$ iss. 607 


\title{
Au-delà de l'évolution technologique : réflexion muséologique pour des cyberexpositions conséquentes et particularisées
}

\author{
Eric Langlois \\ Université du Québec en Outaouais - Canada
}

Il y a l'outil, la fonction de l'outil et le projet qui anime le sujet qui le conçoit ou qui en fait usage. L'outil technique, voire la machine, évolue implicitement par l'usage que l'on en fait et parce que l'expérience inhérente à celui-ci nous amène à le perfectionner. Il doit pouvoir convenir à nos desseins et, par là, nous représenter :

[...] la relation physique entre les objets et les sujets fait culture. Le terme de culture matérielle ne se réduirait donc pas aux objets matériels, mais intégrerait la relation entre les sujets et les objets. (Julien \& Rosselin, 2005, p. 6)

II s'agit dès lors de la dynamique matérielle et sociale « objet-sujetculture ».

Si la recherche et le développement sont les moteurs scientifique et économique de l'évolution technologique, l'expérience que l'on fait de nos divers objets techniques est quant à elle vectrice d'exigences. Les uns poussent les limites des qualités matérielles et fonctionnelles de leur production matérielle, les autres l'utilisent et raffinent en conséquence leurs attentes. Cette évolution, comme un continuum prospère, engendre une cadence. Notons que les concepts d' "évolution technologique " et de "représentation culturelle » sont étroitement liés. Si usage et développement technologique se stimulent réciproquement, il ne faut pas se méprendre sur l'objectif de l'entreprise : l'outil est au service de l'homme, et non l'inverse.

Il est primordial, dans le domaine qui nous intéresse - soit les formes numériques de diffusion muséale et, par extension, la cybermuséologie $^{76}$-, de ne pas confondre les moyens avec l'intention.

\footnotetext{
[...] le savoir se trouve alors aspiré par la connaissance de la technique, comme assigné à résidence. II institue ainsi la technique comme dispositif central des mutations. En outre, une telle démarche relègue à l'arrière-plan une multiplicité de facteurs économiques, sociaux, politiques, institutionnels, propres au domaine envisagé. Or le milieu d'accueil des propositions technologiques, le musée en l'occurrence, reste décisif, il interprète et traduit l'apport spécifique des nouveaux outils en fonctions des logiques qui l'animent. (Welger-Barboza, 2001, p. 11)
}

\footnotetext{
${ }^{76}$ Notons brièvement que le cybermusée, tel que nous pouvons l'observer aujourd'hui et si divers soit-il dans ses actualisations, a été rendu possible grâce à l'accès public d'Internet (1993-1994) et à la numérisation massive des collections (amorcée dans les années 1990 et ayant culminé au tournant du millénaire). En parallèle, les productions hypermédiatiques muséales hors-ligne, tels les CD-Roms et les DVD-Roms, ont eu leurs heures de gloire, maintenant révolues. Celles-ci ne présentent plus d'avantages médiatiques concurrentiels par rapport aux productions cybermédiatiques actuelles. Évidemment, si les musées ont été à la base des productions cybermuséales, cela a également eu des impacts dans la réalisation de leur mission. Ils doivent maintenant aussi s'accomplir de manière extra-muros. L'accessibilité et la démocratisation du patrimoine, tout comme la participation et la collaboration des publics, en sont les éléments moteurs.
} 
Certes, le média est éminemment technologique, mais il convient de convoquer les savoirs et les savoir-faire de la muséologie. L'approche requiert une mise en perspective, un recul. Quelle posture adopter face aux nouvelles possibilités technologiques? À ce propos, Julien et Rosselin (2005) nous rappellent les mots du chercheur français Pierre Lemonnier (1996) au sujet de la signification de nos gestes techniques.

[...] s'interroger sur l'adéquation des actions techniques aux
effets physiques que l'on cherche à atteindre à travers elles
- c'est-à-dire sur leur efficacité relative - est une manière
simple d'entrer de plain-pied dans l'univers des
représentations sociales afférentes à ces actions.
(Lemonnier, 1996, p. $22-23$ cité dans Julien \& Rosselin,
2005, p. 48)

Les représentations sociales, dans le contexte du cybermusée comme objet médiatique de culture matérielle, se manifestent sur deux plans d'une même proposition identitaire, d'une intention culturelle : 1) la médiatisation - soit la production cybermédiatique comme trace de l'action humaine portée par l'objet (l'intention d'un projet révélée par son articulation, sa structure) ; 2) la médiation visuelle - soit les objets donnés à voir par l'image et la manière dont ils le sont, et ainsi leurs impacts (effets) expérientiels, cognitifs et affectifs $^{77}$.

Maintenant, en appliquant l'esprit de Lemonnier à la culture matérielle, tentons cette proposition : quel geste pour quel effet, et quel effet pour quelle efficacité relative de telle représentation sociale? Inversons maintenant celle-ci : quelle représentation sociale en fonction de quelle efficacité relative, découlant de quel effet et donc de quel geste? Cette proposition inversée a l'avantage d'illustrer l'enchaînement empirique de tout projet technologique qui se veut pertinemment sensible : partir de la base, du projet, de l'idée, voire de l'intention, pour ensuite produire conséquemment à ce que l'on veut énoncer. Sémiotiquement, on peut évoquer cela par la question suivante : que veut-on donner à voir et comment le veut-on (effets physiques) et donc, quels sont les donnants à voir (relevant des actions techniques) et pour quelle interprétation (efficacité relative) finale (représentation sociale)? C'est là également toute la dynamique peircienne. Rien de nouveau : toutefois cette évidence n'est pas commune chez les producteurs de contenus numériques. Pourtant, au sein de la dynamique de nos divers systèmes cybermédiatiques mondialisants - et les musées en font partie -, il est d'autant plus pertinent de donner à apprécier des mises en valeur spécifiquement déployées pour des cultures matérielles et immatérielles données. II ne s'agit donc pas de mettre en valeur la technologie: son efficience parlera d'elle-même pour elle-même. Le fameux énoncé "Le message, c'est le médium » (McLuhan, 1993) peut encore une fois être discuté.

Ce texte n'est pas un plaidoyer contre l'intégration et l'usage des technologies de l'heure, voire de demain. Cette évolution est heureuse et elle nous offre toujours de nouvelles possibilités, de nouveaux effets pour montrer et pour dire. Toutefois, le développement efficient et éclairé des pratiques muséales

\footnotetext{
${ }^{77}$ Ces types d'impact découlent d'une mobilisation de la typologie DICAS d'Henry Jacques et de Jocelyne Cormier (2006). Les types d'objectifs proposés par les auteurs peuvent être également entendus comme impacts recherchés. Bien que relevant du champ de l'éducation, cette typologie présente des qualités adaptées au domaine qui nous intéresse.
} 
numériques, à tout le moins en ce qui concerne la diffusion cybermuséale, est l'essence même d'une hypothétique cybermuséologie médiatiquement conséquente et particularisée. Mais qu'est-ce que cette proposition? Ce texte tente d'y répondre en posant les bases réflexives. Produire une cyberexposition, pour ne pas dire la fabriquer, c'est aussi pousser l'efficacité du geste technique né d'un projet, et par là perfectionner un dispositif qui prétend exposer.

\section{Réflexions sémantiques et considérations ontologiques}

\section{Cyber}

Qu'est-ce qu'une cyberexposition? Question pertinente, car ce terme, quoique juste, n'est pas usité.

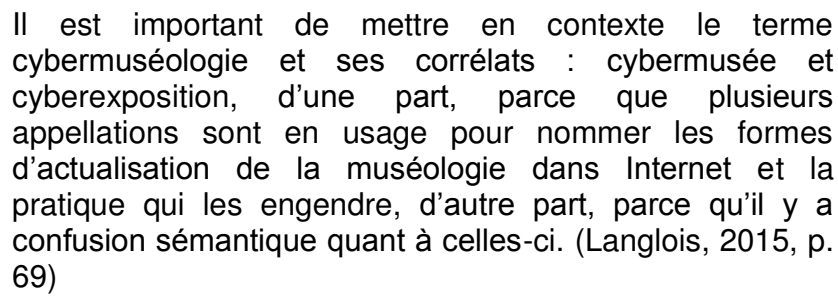

La cyberexposition est un énoncé qui est conçu et réalisé en fonction des fondements pratiques et théoriques relatifs au concept empirique qu'est l'exposition muséale classique et matérielle. Elle est technologiquement numérique; transmise par le réseau qu'est I'Internet; articulée par le protocole qu'est le Web; dotée de caractéristiques hypermédiatiques propres au média ainsi impliqué et, enfin, portée par l'écran, donc implicitement par l'interface.

En adéquation à ces conditions, le préfixe "cyber " indique exactement le contexte de ce qui est en jeu pour une exposition donnée. Il désigne pertinemment le dispositif médiatique qui la donne à voir et qui l'articule.

Brièvement, le préfixe "cyber » (1834) nous vient d'une évolution étymologique issue du besoin d'exprimer "l'étude des moyens de gouvernement ». Appliqué, au $X X^{e}$ siècle, à la relation " être vivant machine ", il reprend ainsi, en 1948, le sens du terme angloaméricain cybernetics. Sous l'égide de cette acception américaine, il s'applique ensuite, vers 1993, à l'automatisme informatique, voire à la robotique, pour ainsi désigner des "organismes électroniques humanoïdes ». Enfin, depuis le développement de ce que l'on appelait jadis "la Toile » et de son utilisation publique, le préfixe « cyber » s'applique à ce qui est porté par l'Internet (Rey, 1998).

Mais revenons à la muséologie : cyberexposition, exposition en ligne, exposition numérique, exposition virtuelle... Comment s'y retrouver parmi ces termes inscrits dans le sens commun et qui semblent désigner les diverses formes d'actualisation muséale et numérique ? En quoi le préfixe « cyber » est-il plus adéquat?

\section{En ligne}

Bien qu'elle soit moins pragmatique et moins précise que " cyberexposition », la locution adjectivale " en ligne » utilisée dans l'appellation " exposition en ligne » demeure, tant en français qu'en anglais, un synonyme acceptable de on line. Deloche (2007) en 
convient d'ailleurs pour ce qui a trait à "musée en ligne ». On s'entend sur le fait que cette locution indique qu'il y a transmission par réseau. Cependant, pourquoi privilégier la locution adjectivale quand un simple préfixe est tout à fait adéquat?

\section{Numérique}

Quant à la terminologie utilisant le qualificatif « numérique », celle-ci est certes envisageable, dès lors qu'elle désigne globalement ce qui est propre à la condition numérique. Une muséologie numérique embrasse donc ce qui est en ligne (cyber) et ce qui ne l'est pas. Une exposition numérique n'est pas forcément une cyberexposition (elle peut être hors ligne, comme dans le cas des bornes interactives) et, par là, le préfixe privilégié " cyber » demeure donc plus précis pour le cas qui nous intéresse (Langlois, 2015, p. 70).

\section{Virtuel}

Enfin, une mise au point détaillée s'impose au sujet du qualificatif « virtuel ». L'utilisation vernaculaire du terme "virtualité » et de ses corrélats a engendré un brouillard sémantique qui est encore loin de se dissiper. Le qualificatif « virtuel » est-il adéquat pour désigner les objets cybermédiatiques muséaux ?

Afin de mieux saisir certains enjeux pour répondre à cette question de sémantique, il faut faire un détour étymologique: l'adjectif " virtuel» est un emprunt au latin scolastique virtualis "qui n'est qu'en puissance ", lui-même dérivé du latin classique virtus "vertu » (Rey, 1998). La question est complexe. Virtus et virtualis, bien que liés par une évolution étymologique commune, sont tout de même porteurs de sens précis. Ils ont à ce titre des implications sémantiques et philosophiques qui leur sont propres. La virtualité, par son double héritage de sens, interpelle aujourd'hui certains chercheurs qui tentent de contextualiser l'usage que l'on en a fait et l'usage et que l'on devrait en faire.

Entre autres, Bernard Deloche (2001) s'accorde avec l'opinion de Pierre Lévy (2001 [1995]) à ce sujet. Aucun des deux ne semble opérer de distinction de sens entre virtus et virtualis. Ainsi, l'évolution étymologique du terme "virtualité » mènerait à signifier, par celui-ci, ce qui est en puissance et en force.

Le mot virtuel vient du latin médiéval virtualis, lui-même issu de virtus, force, puissance. Dans la philosophie scolastique, est virtuel ce qui existe en puissance et non en acte. Le virtuel tend à s'actualiser, sans être passé cependant à la concrétisation effective ou formelle. (Lévy, 2001 [1995], p. 13)

Quoi d'étonnant alors si l'art ${ }^{78}$ produit des effets ? À ce titre il est pleinement virtuel, le terme de force étant, comme on sait, déjà contenu dans l'étymologie du mot virtuel (du latin " virtus » qui, avant de signifier le courage ou la valeur, soit la force de caractère, désigne simplement la force). (Deloche, 2001, pp. 154-155)

On sait que, loin d'être une invention récente comme le croit souvent le grand public tenté de saluer l'apparition d'un néologisme, le terme de virtuel est ancré dans les racines de la pensée occidentale. II vient du latin virtus, qui signifie la puissance ou la capacité à faire quelque chose (Aristote). Ainsi compris, le virtuel ne désigne ni l'irréel ni le

\footnotetext{
${ }^{78}$ Deloche entend l'art - dans les contextes de l'esthétique, du muséal et du virtuel comme le fait de « montrer le sensible par un artefact » $(2001$, p. 17).
} 
numérique ni l'image de synthèse. II n'est que du réel « en puissance d'être actualisé » [...]. (Deloche, 2007, p. 162)

Sommairement, on peut établir que Deloche considère le " musée virtuel » comme une problématique. C'est le grand projet conceptuel du " muséal ». Des solutions créatives concurrentes peuvent en découler. II y en aurait tout un ensemble pour l'actualisation muséale de la mémoire collective ${ }^{79}$. Ces solutions seraient initialement indéterminées, mais le deviendraient à la suite d'une problématisation. Ainsi, il serait permis d'admettre qu'un cybermusée est une des formes actualisées du "musée virtuel ${ }^{80}$. Le cybermusée n'est pas un " musée virtuel ", il est une solution à celui-ci parmi d'autres. Dans ce contexte sémantique et étymologique, " cybermusée » et «musée virtuel » ne peuvent donc pas être entendus comme synonymes, car l'un est une actualisation (solution) de l'autre (problématique). Une fois de plus, le préfixe " cyber" demeure plus adéquat quant à la réalité médiatique qui nous concerne. (Langlois, 2015, p. 70)

Par ailleurs, Denis Berthier (2007) semble quant à lui opposer le sens de virtus au sens de virtualis. II met en perspective les acceptions du terme "virtuel », relatives à la latence, au possible, voire à l'éventuel, par rapport au fait de rendre une équivalence, les qualités sensibles d'une chose, ses effets perceptibles. S'il admet lui aussi le sens de virtualis comme évidemment opposé à ce qui est actuel, il met cependant en lumière une distinction à opérer quant à notre relation au réel. II se sert ainsi d'une logique sémio-cognitive qui induit une pragmatique ontologique: le sujet perçoit " réellement », par la vue, ce qui porte les effets (virtus) d'une réalité tangible et, en ce sens, il ne semble pas y avoir de distinction à opérer entre la perception d'un reflet ou d'une image d'un objet et celle de l'objet lui-même. En mobilisant le sens " virtus-vertu » dans ce qui a trait à l'expérience réelle, il amalgame ainsi le phénomène de la perception visuelle sensible à la virtualité et ainsi, donc, le virtuel au réel.

Finalement, tant que les conditions d'observation sont satisfaites, rien ne permet de distinguer visuellement le reflet de l'objet réel. II n'en va évidemment pas de même dans les autres modalités sensorielles. (Berthier, 2007, p. 2)

Or, est-ce que des conditions d'observation humainement adéquates rendent plus légitime, d'un point de vue ontologique, l'assimilation entre les effets perceptibles d'une chose - à savoir son reflet ou son image - et la chose elle-même? Si l'expérience de la vue est certes une réalité pour le sujet, il n'en demeure pas moins que celui-ci est conscient de ce qu'il regarde. Le sujet placé devant un miroir ou un écran numérique sait bien qu'il est devant un dispositif qui lui permet de se voir ou de voir une image donnée. Quand bien même l'image génère des effets performants eu égard à une réception conditionnée d'un sujet fort volontaire, il n'y a pas de leurre possible ${ }^{81}$. Si le

\footnotetext{
${ }^{79}$ Deloche énumère les solutions suivantes : musée institutionnel, musée de collection privée, musée de la mobilité, musée sans collections, musée inventaire, musée imaginaire $(2007$, p. 163).

${ }^{80}$ Pierre Lévy (2001 [1995], p. 13) comme Bernard Deloche (2001, p. 148) affirme que le virtuel s'oppose à l'actuel et non au réel : en ce sens, "virtualité » et « actualité » seraient deux manières d'être différentes.

${ }^{81}$ Le lecteur intéressé par les théories esthétiques relatives au potentiel d'effet de l'image et au processus de réception de celle-ci, notamment les théories des protagonistes de l'école de Constance et de leurs successeurs, est convié à lire Deloche (2001:26-30).
} 
cybermusée et la cyberexposition arrivaient à rendre perceptible par l'image une substitution (un semblable sensible) du musée ou de l'exposition physique, il n'en demeurerait pas moins que le dispositif serait saillant. C'est justement cet entendement infertile - confondre l'expérience réelle et la réalité d'une chose - qui a fait craindre l'altérité, voire la disparition du musée physique par l'arrivée du cybermusée, une appréhension engendrée par une assimilation médiatique stérile. Ainsi, en tentant d'amalgamer sans discernement les modalités du musée physique à celles du cybermusée, certains confondent également « les vraies choses » et les objets vecteurs de vraisemblance $^{82}$. Le cybermusée n'est pas le musée physique et l'inverse est aussi vrai : ils n'impliquent pas les mêmes composantes, conditions et caractéristiques médiatiques. II convient plutôt de les envisager en complémentarité. Conséquemment, le cybermusée doit donner à voir par les moyens dont il dispose comme média, c'est-àdire par ses qualités hypermédiatiques et, sur ce plan, sa structure est tout à fait perfectible.

En somme, pour toutes les raisons données supra, le qualificatif « virtuel " appliqué au musée, tant dans le sens de virtualis que de virtus, ne peut adéquatement désigner le cybermusée. D'ailleurs, comme nous l'avons explicité, le concept de "virtualité » mobilise plusieurs considérations empiriques et philosophiques. Celles-ci ouvrent sur des problématiques qui englobent, mais dépassent également la réalité de l'occurrence médiatique qui nous intéresse. C'est une raison de plus pour légitimer l'efficacité et l'adéquation de l'appellation «cybermusée».

\section{La portée de l'exposition : quelques considérations}

Avant d'examiner comment exposer par le cybermédia, portons un bref regard sur ce que le geste d'exposer implique. II est évidemment possible d'isoler de multiples considérations à ce sujet, d'ailleurs abondamment documentées ${ }^{83}$. Qu'est-ce que devrait être une exposition ? Qu'est-ce qu'implique l'action d'exposer ? Ces questions demeurent d'actualité, ne serait-ce qu'en raison de l'éclatement des formes de médiatisation actuelles. L'objectif ici est de consolider, en guise de rappel et aux fins de cet article, ce qui est communément admis.

Les différentes acceptions du terme " exposition » renvoient à celleci comme : 1) action; 2) ensemble; 3) lieu; 4) résultat. De fait, il y a le geste d'exposer, la réunion des objets qui le sont, l'espace qui y participe implicitement et, enfin, la finalité envisagée comme occurrence médiatique à apprécier. Cet enchevêtrement n'est pas hermétique et il convient de percevoir celui-ci comme une dynamique.

\section{Action}

D'une part, l'action d'exposer (montrer, présenter, démontrer, expliquer) implique un projet initial (un dessein esthétique, didactique, scientifique et globalement culturel), un processus

\footnotetext{
82 Dans le contexte du musée classique traditionnel comme système de communication et au sujet des " vraies choses » et de la vraisemblance, notamment par le fac-similé et l'image, voir Duncan Cameron (1992 [1968] : 259-262).

${ }^{33}$ Le texte proposé par A. Desvallées, M. Schärer et N. Drouguet, sous l'entrée "Exposition", du Dictionnaire encyclopédique de la muséologie, présente une diversité de points de vue sur l'exposition, diversité qui prend appui sur les travaux de multiples auteurs (Desvallées \& Mairesse, 2011).
} 
théorique et pratique (l'articulation intellectuelle et sensible de la proposition et sa concrétisation), des moyens (matériels et numériques) et, enfin, des producteurs (équipes scientifique, muséographique, scénographique et de l'action culturelle). II existe autant d'approches formelles et conceptuelles que de projets. La preuve en est les multiples typologies pouvant être associées aux stratégies d'exposition.

\section{Ensemble}

D'autre part, l'ensemble constitué par l'exposition comporte des expôts (musealias, substituts, matériels expographiques, outils d'information comme les éléments interactifs et multimédias, composantes de signalisation). Ainsi, certains expôts sont de "vraies choses ", tandis que d'autres en font la suppléance ou les portent et les supportent, les expliquent et les mettent en contexte. Duncan Cameron (1992 [1968], pp. 263-264) désigne ces occurrences médiatiques de l'exposition physique comme étant respectivement les médias non verbaux (soit les "vraies choses", les musealias) et les médias subsidiaires ou secondaires (tels les étiquettes, les narrations, les diagrammes, la photographie, la vidéo). Quels que soient ces musealias, quels sont leur contexte d'origine et leur portée actuelle? On veut circonscrire et communiquer le sens des objets : on vise ainsi la connotation et la dénotation. On veut mettre en valeur ce qui est donné à voir ${ }^{84}$. Ce groupement intentionnel est signifiant, tant pour ses parties que pour son tout. La logique du discours porté est inhérente à la dynamique relationnelle de l'ensemble et l'ensemble sert la logique communicationnelle qui en résulte. Les producteurs d'une exposition élaborent ainsi un groupement qu'ils espèrent significatif.

[...] le sens d'une exposition ne relève pas tant des objets présentés que des jeux de relations qu'ils suscitent, toute la difficulté consiste à qualifier aussi précisément que possible ces relations, à les décrire et à les évaluer. (Glicenstein, 2009, p. 85)

\section{Lieu}

Par ailleurs, l'exposition comme lieu induit un contexte géographique et symbolique (un musée, une ville, un pays, une culture énonciatrice, tout comme un patrimoine exposé). Elle est un espace dédié et signifiant et, à ce titre, elle sert la proposition médiatique et en fait partie. Elle est un territoire spatial dont les qualités et contraintes physiques influencent la mise en valeur des expôts (de là les aspects expographiques et ergonomiques) et en cela elle conduit à diverses possibilités d'ordonnancement, voire d'articulation des objets donnés à voir, et donc à différentes possibilités de parcours de visite. Le lieu envisagé influe aussi sur la mise en ambiance et celleci doit l'investir dans l'esprit de l'exposition. De plus, dans le cadre de stratégie permanente, temporaire ou itinérante, l'exposition influe sur

\footnotetext{
${ }^{84}$ L'expression " mettre en valeur » est régulièrement employée tant à l'oral qu'à l'écrit. Si elle est fortement présente au Québec, elle l'est aussi en France, quoique dans une moindre mesure. La littérature qui tente de la définir est quasi inexistante. En s'accommodant de la doxa muséale, on peut constater que plusieurs muséologues l'utilisent pour signifier la mise en œuvre de conditions optimales aux fins de la valorisation esthétique et scientifique d'un objet, que ce soit par des moyens matériels ou des actions. Cela rend (intrinsèque) ou associe (extrinsèque) des valeurs à un objet donné, et ce, tant pour ses qualités matérielles que symboliques. Dans les faits, il s'agit d'une expression « valise » qui est plus au moins circonscrite. Enfin, si certains considèrent la mise en valeur et l'interprétation comme étant des synonymes, d'autres envisagent l'interprétation comme une des actions possibles de la mise en valeur. Soulignons que la valorisation, comme processus, est surtout liée au champ des patrimoines.
} 
la relation "lieu-exposition »: lieu investi à long terme par une même exposition; lieu investi à court terme par diverses expositions; lieux multiples investis par une même exposition, alors adaptée à ceux-ci.

\title{
Résultat
}

Enfin, l'exposition comme résultat de l'action, de l'ensemble et du lieu impliqués est en soi une proposition médiatique dotée d'un langage composite transmis par diverses modalités. L'exposition est un média qui en inclut d'autres et, à ce titre, elle s'inscrit dans une dynamique communicationnelle. Elle est une proposition sensible dont le visiteur fait "l'expérience », que celle-ci soit individuelle ou sociale. On veut mettre des visiteurs en relation avec des expôts et on veut le faire par des modalités que ceux-ci devront impérativement appréhender. C'est cette expérience comme dynamique communicationnelle qui induit un résultat à géométrie variable: une finalité toujours subjective qui, en bout de piste, est déterminée par le visiteur. Celui-ci reçoit et interprète en effet la proposition du monde muséal - l'exposition - dans le cadre précis de sa propre réalité : projet initial, processus, moyens et producteurs, tout cela aura permis, dans une mesure relative, de donner à voir, à apprécier, à apprendre et à comprendre.

\begin{abstract}
L'exposition ne peut donc jamais se réduire, uniquement et directement, à un simple dispositif instrumental mettant en relation le visiteur avec les choses exposées. Son fonctionnement implique (i) une activité de compréhension de la part du visiteur; (ii) que cette activité de compréhension se déroule dans un contexte communicationnel, au sens où le producteur de l'exposition y a nécessairement mis des indications sur la manière dont le visiteur peut arriver à cette compréhension. Cette double condition revient à reconnaître que l'activité du visiteur est certes, au moins partiellement, cognitive (premier point), mais surtout (second point) que cette activité vise d'abord à saisir la manière dont l'exposition répond à l'intention de le faire accéder à l'objet. (Davallon, 1999, p. 17)
\end{abstract}

Éminemment subjective, l'interprétation du visiteur est également marquée par des stratégies interprétatives et pédagogiques que les producteurs mettent souvent à contribution pour encadrer l'expérience de celui-ci. C'est cette même expérience toute relative de l'exposition qui fait l'objet d'analyses méthodiques par l'évaluation muséale. Qui plus est, on incite le visiteur à s'exprimer et à commenter son expérience.

\section{Exposer par le cybermédia}

Nous y sommes. La cyberexposition est-elle une proposition médiatique qui est en phase avec les considérations propres à l'exposition classique et matérielle? Pour comprendre en quoi le fait d'exposer conséquemment par le cybermédia demande à prendre en compte les particularités intrinsèques de celui-ci, tentons une mise en parallèle $e^{85}$.

\section{Cyberexposition comme « action »}

\footnotetext{
${ }^{85}$ L'auteur met ici à profit ses travaux de recherche doctorale sur l'interface Web comme sémiophore cybermuséal (Langlois, 2013).
} 
Soulignons d'abord que la cyberexposition reprend, dans une large mesure, les mêmes actions que celles induites par le fait d'exposer matériellement. On veut montrer des objets. Cependant, on ne le fait pas en les présentant comme signes-objets présents, voire comme de " vraies choses ", mais plutôt en les représentant par l'image qui, elle, est le signe du sujet qu'est l'objet absent. C'est la vraisemblance de Cameron (1992 [1968], pp. 259-262). La nature de l'image est presque toujours indiciaire (photographique), car la fidélité au sujet est de mise pour sa juste représentation. Ainsi, on peut tout à fait qualifier cette opération de substitution. S'ajoutent au geste de représenter par l'image ceux d'expliquer et de démontrer : par le texte, par d'autres images fixes ou cinétiques, par le son. Pour utiliser la terminologie de Duncan Cameron dans le contexte de la cyberexposition : ce sont des médias subsidiaires qui sont relatifs à des médias substitutifs. C'est ce que Jay Lemke (2002) appelle la multimodalité : plusieurs modalités sont ainsi convoquées comme agents signifiants. Outre le fait d'identifier les objets représentés par des légendes ou des fiches signalétiques (connotation), on veut également mettre ceux-ci en contexte (dénotation) par l'explication. De plus, dans certains cas, et en fonction de la nature des objets impliqués, on veut démontrer certains principes ou phénomènes qui sont relatifs à l'objet représenté. Ce sont les " kinétifacts " de Cameron (1992 [1968], p. 262). On veut également rendre compte de certains témoignages. Jusque-là, outre la nécessaire représentation par l'image, le projet muséal relève des mêmes intentions: on vise la mise en contexte et la mise en valeur. Finalement, il faut également prendre en compte les hyperliens qui sont implicites à toute production hypermédiatique. Ils opèrent, entre les diverses occurrences, des modalités qui servent à représenter, à expliquer et à démontrer. Ils induisent ipso facto du sens par les mises en relation qui en résultent. C'est ce qu'il convient de nommer l'hypermodalité (Lemke, 2002). Nous avons vu qu'au sein de l'exposition classique matérielle des liens entre les expôts opèrent aussi pour l'ensemble du discours. Simplement, dans le cas de la cyberexposition, ces liens sont implicites parce qu'ils s'inscrivent dans le fonctionnement du dispositif hypermédiatique.

En somme, il en va ainsi de l'action d'exposer par le cybermédia :

1) le dessein initial peut être de l'ordre de l'esthétique, de la didactique, du scientifique et globalement du culturel;

2) des processus théoriques et pratiques visant l'articulation intellectuelle (les contenus) et sensible (l'interface portée par l'écran et qui se veut fonctionnelle et signifiante) y sont impliqués;

3) la cyberexposition nécessite des moyens expographiques (numériques); et finalement,

4) elle nécessite des producteurs, c'est-à-dire une équipe scientifique, des muséologues, d'autres acteurs de l'éducation muséale et des services culturels issus de l'instance muséale énonciatrice ainsi qu'une équipe de production hypermédiatique souvent composée de prestataires.

\section{Cyberexposition comme " ensemble "}

Que sont concrètement les types d'expôts d'une cyberexposition?

1. Le matériel cyberexpographique - On peut affirmer que ceux-ci sont de nature numérique et que cela va de soi, mais ce serait oublier toute la matérialité du dispositif : une cyberexposition requiert un appareillage matériel qui comprend l'indispensable écran qui rend visible l'interface (tactile ou non), l'ordinateur (mobile ou non) et les périphériques d'interaction (souris et clavier) encore régulièrement nécessaires (malgré l'arrivée des écrans tactiles). 
2. Les éléments portés par l'interface - Une cyberexposition est un ensemble porté et articulé par l'outil d'information sensible, interactif et multimédia qu'est l'interface. Celle-ci se décline en divers éléments qui sont à considérer comme expôts (Langlois, 2013) ${ }^{86}$.

a. Les éléments d'ambiance - Principalement le graphisme comme stratégie esthétique pour composer l'atmosphère de l'interface en fonction de la thématique impliquée.

b. Les éléments de contenus - Des images pour représenter les objets $^{87}$, et des composants multimodaux d'information connotatifs et dénotatifs (textes, images, sons) pour démontrer, expliquer et ainsi porter le discours.

c. Les éléments fonctionnels - Une signalétique opérationnelle, régissant la navigation, issue d'une structure essentiellement composée de "cliquables " tels que les boutons, les icônes, les menus, les hyperliens.

\section{Cyberexposition comme " lieu »}

Le lieu de la cyberexposition est le site Web dont les coordonnées sont identifiées par l'adresse URL. En cela, il n'est pas un lieu géographique, mais un cyberlieu. La nature géographique et symbolique de ce lieu est induite par l'énonciateur. Celui-ci (le musée) relève bel et bien d'une culture énonciatrice et d'un patrimoine qui lui est propre et qui peut être situé géographiquement. On peut aussi affirmer qu'un cyberlieu est une réalité symbolique parce que non tangible (si ce n'est par sa matérialité induite par l'écran) et parce que ce type de lieu relève, d'une certaine façon, de la métaphore (le terme «site» associé au Web). Par ailleurs, la cyberexposition est un lieu dédié et signifiant : le site Web, par ses interfaces, est un espace médiatique qui sert une proposition. Parce que ce type de lieu implique un dispositif hypermédiatique, il impose comme espace à investir des qualités et des contraintes qui sont le propre de son fonctionnement. Le graphisme et l'ergonomie, voire la cyberexpographie, doivent en être conséquents. Diverses possibilités d'ordonnancement des expôts numériques sont possibles et exponentielles. La configuration d'un parcours, soit la navigation propre à chaque site Web, doit être tout aussi pertinente à l'articulation d'un discours préalablement scénarisé qu'à une architecture Web pragmatique.

Enfin, la cyberexposition en tant que lieu est soumise à différentes stratégies comme occurrence médiatique. Le producteur, le musée comme occurrence énonciatrice, optera pour l'une ou l'autre des diverses approches pour rendre accessible sa proposition ${ }^{88}$ :

1. la cyberexposition indépendante (elle n'a aucun lien avec une exposition physique);

2. la cyberexposition complémentaire précédente (mise en ligne avant l'ouverture de l'exposition physique);

3. la cyberexposition complémentaire simultanée (mis en ligne dans le même temps que l'exposition physique); et

4. la cyberexposition complémentaire ultérieure (comme proposition prolongeant et pérennisant l'exposition physique).

\footnotetext{
${ }^{86}$ Les termes " atmosphère », " discours » et "structure » sont ici littéralement utilisés en référence aux différents niveaux de signification de l'interface, tels qu'ils sont proposés par Pignier, Drouillat et Fontanille (2005).

${ }^{87}$ Les musealias n'étant pas présents afin d'être exposés, on les représente donc par l'image numérique pour les substituer (sauf dans les cas où une cyberexposition présente des artefacts ou des œuvres qui sont par essence numériques, et qui seraient alors à considérer conséquemment comme musealias numériques).

${ }_{88}$ L'auteur utilise ici une typologie relative aux stratégies de cyberdiffusion qu'il a élaborée en 2003 dans le cadre de ses travaux de recherche et d'enseignement.
} 
Cette déclinaison stratégique n'est pas hermétique et l'ordre proposé n'est pas en soi une prescription. Elle permet cependant de saisir les relations que pourrait entretenir la cyberexposition avec ce qui est exposé au musée physique, qu'il s'agisse d'une exposition permanente, temporaire ou itinérante.

Cyberexposition comme « résultat »

La cyberexposition peut finalement être considérée comme le résultat de l'action, de l'ensemble et du lieu. Ces considérations sont intrinsèquement contributives de l'exposition tant cybermédiatique que physique. Globalement, cette mise en parallèle autorise les rapprochements suivants :

1. toutes deux sont dotées d'un langage composite (la multimodalité);

2. elles s'inscrivent respectivement et éminemment dans des dynamiques communicationnelles et sensibles (cependant, le sens du toucher n'est pas communément convoqué par la cyberexposition, mais souvent, il ne l'est pas davantage au musée physique);

3. elles relèvent toutes deux de l'expérience (il y a bel et bien un individu qui en fait l'usage et qui expérimente une réalité) et celle-ci peut être soit individuelle soit sociale (la cyberexposition, malgré l'inclusion des médias sociaux et a contrario de l'exposition physique, relève davantage de l'expérience individuelle);

4. dans les deux cas de figure, le résultat est toujours subjectif parce que soumis à la réception et à l'interprétation d'un individu dans le cadre de sa réalité;

5. des stratégies interprétatives et pédagogiques y sont dans les deux cas mises à contribution;

6. elles font l'objet d'une évaluation muséale; et

7. la cyberexposition et l'exposition physique prévoient toutes deux que le visiteur s'exprime (le plus souvent maintenant par les médias sociaux).

Enfin, est-il possible d'exposer par le cybermédia? Le présent exercice autorise ici une réponse : il est certainement possible de conclure que le contexte cybermédiatique permet l'exposition dans le cadre du champ muséal. La cyberexposition ne relève pas du catalogue en ligne : le dessein d'exposer se transpose pertinemment au cybermédia et il convient d'en prendre acte. Certes, des différences ontologiques découlent de cette transposition. Cependant, une fois ce constat mis en lumière, ces différences sontelles si fondamentales? Nous ne sommes pas dans le même registre médiatique et l'une n'est pas directement concurrente de l'autre. S'il faut entrevoir tout cela par la complémentarité, il convient aussi de considérer adéquatement les particularités du cybermédia pour être en mesure de concevoir et de produire des cyberexpositions de façon conséquente. II faut mettre à profit le cybermédia pour ce qu'il est et pour ce qu'il permet.

\section{Des cyberexpositions particularisées}

Alors, qu'en est-il de cette proposition de cyberexpositions particularisées? Mettre à profit le cybermédia pour ce qu'il permet, certes, mais encore faut-il le faire pertinemment en fonction des objets que l'on veut représenter et mettre en valeur. Actuellement, les cyberexpositions proposent bien souvent des énoncés hypermédiatiques formatés par une façon de faire qui relève d'une 
quasi-neutralité significative. En ce sens, elles sont sous-exploitées. La construction spatiale de l'interface donne simplement à voir une image de l'objet, accompagnée d'une fiche signalétique et d'un court texte de mise en contexte. Cette formule de médiatisation est devenue la norme, et la tendance à l'épuration des interfaces des dernières années y a contribué. Si cette épuration a permis un meilleur contrôle ergonomique du média, elle a toutefois induit des approches cyberexpographiques moins particularisées. L'arrivée d'une réalité médiatique multiplate-forme, par sa complexité de compatibilité en regard des différents formats d'écrans, a aussi fait pencher la balance du côté des stratégies uniformisantes.

Qu'en est-il d'ailleurs, dans le contexte de la cyberexposition, des questions de stratégies de mise en valeur en fonction d'un objet qui serait a priori doté par lui-même et pour lui-même d'un pouvoir de transmission d'informations: l'objet «parlant» ou l'objet " ventriloque » (Clément, 1983)? Convenons que les musées ont depuis longtemps compté sur leurs pratiques d'exposition pour faire « parler » leurs objets. "En tant que système de communication, le musée dépend alors du langage non verbal des objets et des phénomènes observables » (Cameron, 1992 [1968], p. 260) Or, dans le cas qui nous intéresse, l'objet est absent. II est suppléé par l'image. Si certains ont la conviction que l'objet peut " parler » de luimême, que dire de son image? D'autre part, si la mise en contexte de celui-ci s'avère nécessaire, comment la rendre opérationnelle à partir d'une représentation?

Tentons alors de dégager des pistes de réflexion quant à la particularisation des cyberexpositions en abordant certains éléments sous trois axes : 1) les types d'objets muséaux et leurs conditions ; 2) les sciences qu'ils convoquent ; 3 ) les approches disciplinaires de mise en exposition.

\section{Types d'objets muséaux}

On peut d'emblée affirmer que les cyberexpositions comportent des médiations $^{89}$ des types d'objets suivants: artefacts, écofacts ${ }^{90}$, $œ u v r e s$. Détaillons les conditions qui leur sont intrinsèques ${ }^{91}$.

- D'abord, les artefacts (matériels ou numériques) induisent différentes conditions qui leur sont propres. On peut globalement les inventorier ainsi: statiques, articulés, animés, interactifs, événementiels, documentaires.

- Ensuite, les écofacts dans leurs diverses conditions (qu'ils soient humains, animaux, végétaux, minéraux ou phénomènes en soi) peuvent se décliner comme suit : statiques, vivants, événementiels, documentaires.

- Enfin, les différentes conditions des œuvres (matérielles, numériques ou hybrides) peuvent faire l'objet de cette déclinaison: statiques, cinétiques, animées, interactives, sonores, événementielles.

\footnotetext{
${ }^{89}$ Rappelons, encore une fois, que le terme « médiation » est ici entendu dans le sens de consignation médiatique. Par exemple, on peut consigner l'image d'un objet par la photographie (Saouter, 2011). II convient alors de le différencier de la médiation muséale.

${ }_{90}$ Le terme " écofact », initialement utilisé dans le champ de l'archéologie, est ici utilisé pour qualifier les objets qui sont produits par la nature, a contrario des artefacts qui sont produits par l'homme. Laurier Lacroix l'utilise notamment quant aux types d'objets muséaux (Lacroix, 2002).

${ }^{91}$ La typologie utilisée ici a été élaborée par l'auteur et a fait l'objet de sa conférence De la matérialité au numérique : typologie des situations médiatiques culturelles relatives au champ muséal prononcée dans le cadre de l'Université d'été de l'Université Laval, Musée de la civilisation, Québec, CANADA, juin 2012. Elle évolue toujours en fonction de divers travaux de recherche.
} 
Quoi qu'il en soit, tous ces types d'objets, par leurs conditions, qu'ils soient inertes ou mus par des dynamiques physiques, biologiques, mécaniques, chimiques, électroniques, analogiques ou numériques, ont des particularités qui leur sont propres.

Selon leurs conditions, il convient de les donner à voir par l'image, de façon à rendre compte de leur matérialité (connotation). Dans tous les cas, on souhaite optimiser la qualité (résolution) de l'image bidimensionnelle pour permettre un rapprochement visuel et ainsi donner à voir au plus près les indices de la réalité matérielle des objets. En ce sens, l'image tridimensionnelle ira plus loin, permettant de «manipuler » l'objet dans toutes ses dimensions. L'image en mouvement (vidéo) permettra de rendre au mieux la réalité effective des objets qui se déploient dans le temps et dans l'espace. De plus, lors des opérations de consignation et de traitement des images ainsi obtenues, il convient de s'assurer de ne pas infliger de biais visuels et donc de sens. Déjà, en 1964, Malraux (1996) se préoccupait de travers signifiants que pouvaient engendrer la représentation d'un sujet par la photographie et la juxtaposition des différentes occurrences ainsi obtenues. Les mises en relation d'images d'objet et, ainsi, des champs de la métamorphose, comportent aussi leurs pièges. À l'époque de Malraux et de son musée imaginaire, voire indiciaire, la chromatique était biaisée parce que rabattue en noir et blanc. Les proportions réelles des objets et les rapports d'échelle étaient en outre confinés aux formats des clichés photo. Bien que les choses aient évolué depuis, on veillera tout de même à opter pour le procédé de consignation - et donc pour le type d'images - qui rendra au mieux la réalité de l'objet en fonction de la condition de celui-ci. II s'avère aussi important de s'interroger sur nos objectifs de consignation. Utilise-t-on l'image d'objet pour des fins de substitution, de témoignage ou de démonstration?

En somme, des particularisations en termes de médiation s'imposent : un canot en écorce des Premières Nations, un papillon se libérant de sa chrysalide, une œuvre cinétique d'Alexander Calder, Guernica de Picasso, une pièce numismatique... II convient d'y réfléchir.

\section{Sciences convoquées}

Les divers objets muséaux ont été interrogés par les sciences dont ils relèvent et ils le sont toujours. II en va ainsi de la recherche muséale et de la construction de la connaissance relative aux collections. Les artefacts, les écofacts et les œuvres, ainsi documentés, engendrent de multiples contenus informationnels qui peuvent servir autant à la connotation qu'à la dénotation, et ce, aux fins de la mise en valeur et de l'interprétation.

En considérant ne serait-ce que les usuelles informations signalétiques, nous pourrions facilement isoler différentes particularités à mettre en évidence.

Histoire, anthropologie, ethnologie, archéologie, sciences et technologie. Les artefacts sont associés à leurs producteurs, à leurs matériaux, à leurs techniques de fabrication, à l'époque de leur fabrication, à leurs aspects formels, à leurs propriétaires initiaux et subséquents, à leurs fonctions et usages, à leur valeur marchande, à leur mode d'acquisition, à leurs relations avec d'autres objets au sein de certains systèmes, etc.

Sciences naturelles. Les écofacts sont détaillés par leurs noms commun et scientifique, par leur ordre taxonomique, par leur lieu 
d'origine, par l'endroit de leur découverte, par leur habitat, par leur comportement, par leur mode d'acquisition, par leurs relations avec d'autres écofacts, etc.

Arts. Les œuvres sont quant à elles documentées en fonction de leurs auteurs, de leurs techniques et matériaux, de leur support, de leur format, de leurs propriétaires initiaux et subséquents, de leur mode d'acquisition, de leur valeur marchande, de leurs inclusion et signification dans le champ de l'art, etc.

Tout bien considéré, ces sommes d'informations devraient amener le producteur de cyberexposition à utiliser sciemment le cybermédia pour construire des liens de sens entre ces occurrences informatives. De fait, c'est en mettant à profit les bons modes de consignation par l'image en fonction de ce que l'on veut donner à voir de l'objet et en optimisant l'hypermodalité (hyperliens entre l'image, le texte et le son) que l'on peut rendre la cyberexposition plus signifiante. Juxtaposer différentes modalités signifiantes et créer des liens de sens entre elles ne relèvent pas que de la simple « mise en page " d'interfaces. Enfin, la prise de conscience actuelle quant au patrimoine immatériel culturel, qui est nécessairement documenté par l'image ou le son, ne peut-elle pas induire de pertinentes pistes en ce sens? Les contenus scientifiques développés autour de nos objets ne sont pas de simples données de catalogage. Ils sont autant de pistes de scénarisation et d'interprétation pour la cyberexposition.

\section{Approches disciplinaires de mise en exposition}

Finalement, les approches disciplinaires en regard des principes usuels de mise en exposition physique présentent également de bonnes pistes cyberexpographiques. Par la simple observation, il est aisé de constater que des orientations expographiques prévalent en fonction du type de musée concerné. Sans vouloir faire de raccourci méthodologique, certains stéréotypes sont un état de fait. Autorisons-nous certaines généralités.

D'abord, les musées d'art souhaitent laisser toute la place à " l'aura » de l'œuvre. Ils privilégient la délectation, la contemplation, la mise en valeur d'une esthétique.

Les musées d'histoire, d'ethnologie et d'archéologie traitent quant à eux l'objet-témoin comme faire-valoir d'un passé. Ils favorisent le fait et l'effet historique (la reconstitution matérielle ou narrative des contextes d'origine) au sein de stratégies interprétatives très présentes.

Par ailleurs, les musées de sciences naturelles et de sciences et technologies sont d'emblée des vitrines pour les sciences qui les alimentent. L'objet est montré, certes, et la plupart du temps à titre d'exemplaire type. On le présente dans son contexte taxonomique et on souhaite le faire avec le plus d'indices possible quant à son contexte d'origine. On tente également de montrer et d'expliciter les structures et les dynamiques des écosystèmes, comme on tente de démontrer les principes et les lois scientifiques par le phénoménal (Cameron, 1992 [1968], p. 261). En ce sens, divers moyens interactifs (matériels et numériques) sont régulièrement mis à contribution.

Les musées ont développé des approches adaptées à la nature de leurs collections et, en cela, il convient de s'en inspirer. De fait, dans le cadre de la cyberexposition, la délectation, la quête cognitive et l'expérience interactive bénéficient de l'évolution technologique pour 
devenir de plus en plus performantes. II convient, dans le cadre des cyberexpositions, de ne pas dénaturer ces approches, mais de les transposer, voire de les perfectionner en regard de l'interface ellemême, de ce qu'elle donne à voir et de la manière dont elle le fait. L'ensemble de ces pistes de réflexion peut reconduire à produire des cyberexpositions plus particularisées. À l'époque, les contraintes technologiques exigeaient l'ingéniosité des approches. Aujourd'hui, la multitude de possibilités technologiques permet une mise à profit sensible et efficace. Les stratégies hypermédiatiques sont devenues exponentielles.

\section{Réflexions muséologiques pour une cybermuséologie}

Reprenons le premier énoncé de cet article :

Il y a l'outil, la fonction de l'outil et le projet qui anime le sujet qui le conçoit ou qui en fait l'usage. L'outil technique, voire la machine, évolue implicitement par l'usage que l'on en fait et parce que l'expérience inhérente à celui-ci nous amène à le perfectionner.

Pour y arriver, il faut aussi développer une réflexion théorique qui questionne nos pratiques. Meunier et Fourcade (2013) ont récemment interrogé Bernard Deloche à propos de la cybermuséologie: "Pensez-vous qu'il existe aujourd'hui ce que certains appellent la "cybermuséologie"? " Voici un passage significatif de sa réponse :

Il y a effectivement aujourd'hui des travaux consacrés au cybermusée, parfois appelé à tort musée virtuel. Mais s'agit-il vraiment de traités de muséologie spécifiques? Non. Généralement, ils se contentent de décrire de façon plus ou moins admirative les nouvelles techniques [...]. (Meunier \& Fourcade, 2013, p. 61)

Sans prétendre que cet article soit une pierre ajoutée dans l'édification d'une cybermuséologie, l'intention qui le soutient relève cependant d'une volonté d'y contribuer. II expose à ce titre des liens entre muséologie et pratiques hypermédiatiques et des pistes de réflexion visant une nécessaire mise en perspective qui, elle, devra se poursuivre.

Ainsi, se pourrait-il que le cybermusée puisse tendre vers une juste mise en valeur de la matérialité, sans toutefois vouloir se confondre avec elle ? II serait pertinent de cerner des cas d'étude, puis de les passer sous le filtre des intentions de cet article, c'est-à-dire, de les analyser sous le spectre de la particularisation pour y voir des pratiques exemplaires et d'autres prometteuses, d'autres encore qui remettront en question cette proposition. Enfin, il s'agit de voir plus loin que l'outil et de tendre vers une réelle réflexion cybermuséologique.

\section{Références}

Berthier, D. (2007). «Qu'est-ce que le virtuel? » La Jaune et la Rouge, vol. 626 (Juin/Juillet).

Cameron, D. (1992). « Un point de vue : le musée considéré comme système de communication et les implications de ce système dans les programmes éducatifs muséaux. " In A. Desvallées (Ed.), Vagues : une anthologie de la nouvelle muséologie, vol. 1 (pp. 259- 
270). Mâcon/Savigny-le-Temple: Éditions W/ M.N.E.S. ( $1^{\text {re }}$ éd. 1968).

Clément, B. (1983). "Le mythe de l'objet ventriloque. " Dans BarbierBouvet, J.-F. \& Centre Georges-Pompidou (dir.), Histoires d'expo (pp. 37-40). Paris, France: Centre Georges-Pompidou.

Davallon, J. (1999). L'exposition à l'œuvre: stratégies de communication et médiation symbolique. Paris: L'Harmattan.

Deloche, B. (2001). Le musée virtuel : vers une éthique des nouvelles images. Paris: Presses universitaires de France.

Deloche, B. (2007). La nouvelle culture : la mutation des pratiques sociales ordinaires et l'avenir des institutions culturelles. Paris: L'Harmattan.

Desvallées, A., \& Mairesse, A. (Dir.). (2011). Dictionnaire encyclopédique de la muséologie. Paris: Armand Colin.

Glicenstein, J. (2009). L'art: une histoire d'expositions. Paris: Presses universitaires de France.

Julien, M., \& Rosselin, C. (2005). La culture matérielle. Paris: La Découverte.

Henry, J., \& Cormier, J. (2006). Taxonomie de DICAS. Les archives de DICAS. Saint-Jérôme, Canada: Commission scolaire de la Rivièredu-Nord. À partir de http://csrdn.qc.ca/discas/index.html

Lacroix, L. (2002). Les collections muséales au Québec. Société des musées québécois. À partir de http://www.musees.quebec. museum/pdf/mad-lacroix.pdf.

Langlois, E. (2013). L'interface Web est un sémiophore : regard sémiotique sur les conditions et les composantes d'un énoncé pictural fonctionnel. À paraître.

Langlois, E. (2015). « La cybermuséologie et ses nouveaux objets culturels : mise en contexte et étude de cas. " Muséologies: les cahiers d'études supérieures, 8(1), 67-86.

Lemke, J. L. (2002). "Travels in hypermodality. " Visual Communication, $1(3), 299-325$.

Lévy, P. (2001). Qu'est-ce que le virtuel ? Paris: Edition La Découverte (1 $1^{\text {re }}$ éd. 1995).

Malraux, A. (1996). Le musée imaginaire. Paris: Gallimard ( $1^{\text {re }}$ éd. 1965).

McLuhan, M. (1993). Pour comprendre les médias: les prolongements technologiques de l'homme. Saint-Laurent, Québec: Bibliothèque québécoise ( $1^{\text {re }}$ éd. 1968).

Meunier, A., \& Fourcade, M.-B. (2013). «Entretien avec Bernard Deloche. » Dans A. Meunier, M.-B. Fourcade, \& Mariani, A. (Dir), Muséologies : les cahiers d'études supérieures, 6(2), 53-62.

Pignier, N., Drouillat, B., \& Fontanille, J. (2005). Penser le webdesign modèles sémiotiques pour les projets multimédias. Paris: l'Harmattan.

Rey, A. (Dir.). (1998). Virtuel. Dictionnaire historique de la langue française, tome 2. Paris: Dictionnaires Le Robert.

Saouter, C. (2011). Médiation et médiatisation: quelques redéfinitions. Inédit. Montréal.

Welger-Barboza, C. (2001). Le patrimoine à l'ère du document numérique : du musée virtuel au musée médiathèque. Paris: l'Harmattan.

\section{Résumé}

Qu'est-ce qu'une cyberexposition ? Pourquoi utiliser ce terme? Qu'est-ce que le geste d'exposer et est-il possible de l'accomplir par le cybermédia ? Qu'est-ce que cela suppose?

Cet article tente de répondre à ces questions à travers des considérations ontologiques, sémantiques, médiatiques et disciplinaires. Ce faisant, il invite à rendre l'énoncé hypermédiatique muséal perfectible pour tendre vers une plus grande efficacité sensible et communicationnelle. II s'agit en fait de prendre acte de ce qu'il convient de considérer afin de concevoir et de produire des cyberexpositions: 1) médiatiquement conséquentes; et 2) particularisées en fonction des sciences et disciplines que convoque ce qui est exposé. 
À l'opposé des stratégies uniformisantes et minimalistes qui se sont opérées au sein de l'univers cybermuséal des dernières années, l'auteur en appelle à la prise en compte des possibilités hypermédiatiques et à leur mise en œuvre efficiente. Au-delà de l'engouement que suscitent les avancées technologiques, il propose une réflexion sur les pratiques à adopter pour tendre vers le développement d'une réelle cybermuséologie.

Mots clé : cybermusées, communication, cybermuséologie.

\section{Abstract}

Beyond the technological revolution: museological thoughts about consistent and individual cyber exhibitions

What is a cyber-exhibition? Why use this term? What is the act of display, and can it be achieved through cybermedia? What does the process involve?

This article seeks to answer these questions through various ontological, semantic, media and disciplinary considerations. In so doing, it calls for refining the current hypermedia-based museological construct towards a greater effectiveness that is both sensitive and communicational. In essence, the article explores what should be taken into account when developing and producing cyber-exhibitions: 1) that there be a consistent media-oriented focus; and 2) that what is displayed, and how, must reflect the sciences and disciplines involved.

Unlike the standardized and minimalist strategies employed within the cyber-museum world over the past several years, the author encourages enhanced awareness of the possibilities presented by hypermedia, as well as their relevant and effective use. Looking beyond the excitement that greets every technological advance, he invites reflection upon the practices that might be adopted towards developing true cybermuseology.

Key words: cybermuseums, communication, cybermuseology. 